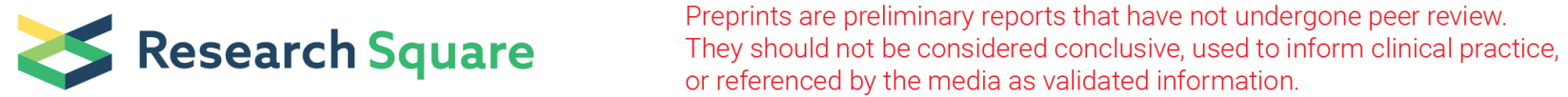

\section{Relationship Between Mean Vancomycin Trough Concentration and Mortality in Critically III Patients: A Multicenter Retrospective Study}

\author{
Yanli Hou \\ Xi'an Jiaotong University Second Affiliated Hospital \\ Jiajia Ren \\ Xi'an Jiaotong University Second Affiliated Hospital \\ Jiamei Li \\ Xi'an Jiaotong University Second Affiliated Hospital \\ Xuting Jin \\ Xi'an Jiaotong University Second Affiliated Hospital \\ Ya Gao \\ Xi'an Jiaotong University Second Affiliated Hospital \\ Ruohan Li \\ Xi'an Jiaotong University Second Affiliated Hospital \\ Jingjing Zhang \\ Xi'an Jiaotong University Second Affiliated Hospital \\ Xiaochuang Wang \\ Xi'an Jiaotong University Second Affiliated Hospital \\ Xinyu Li \\ Xi'an Jiaotong University Second Affiliated Hospital \\ Gang Wang ( $\sim$ gang_wang@xjtu.edu.cn ) \\ Xi'an Jiaotong University Second Affiliated Hospital https://orcid.org/0000-0002-5905-7760
}

\section{Research}

Keywords: vancomycin trough concentration, intensive care unit, mortality, elCU Collaborative Research Database, observational study

Posted Date: February 24th, 2021

DOI: https://doi.org/10.21203/rs.3.rs-232527/v1

License: (c) (i) This work is licensed under a Creative Commons Attribution 4.0 International License. Read Full License 


\section{Abstract}

Background: It remains unclear whether the mean vancomycin trough concentration (VTC) derived from the entire course of therapy is of potential benefit for critically ill patients. This study was conducted to explore the association between mean serum VTC and mortality in intensive care units (ICUs).

Methods: 3,364 adult patients with two or more VTC records after receiving vancomycin therapy in the elCU Collaborative Research Database were included in this multicenter retrospective cohort study. Mean VTC was estimated using all measured VTCs and investigated as a continuous and categorical variable. Patients were categorized into four groups according to mean VTC: $<10,10-15,15-20$, and $>20 \mathrm{mg} / \mathrm{L}$. Multivariable logistic regression and subgroup analyses were performed to investigate the relationship of mean VTC with mortality.

Results: After adjusting for a series of covariates, logistic regression analyses indicated that mean VTC, as a continuous variable, was positively correlated with ICU (odds ratio, 1.042, 95\% confidence interval, [1.017-1.068]) and hospital (1.025 [1.004-1.046]) mortalities. As a categorical variable, mean VTC at 10-15 mg/L failed to reduce ICU mortality (1.512 [0.849-2.694]). Moreover, mean VTCs of 15-20 and >20 mg/L were significantly associated with higher ICU mortality (1.946 [1.106-3.424]; 2.314 [1.296-4.132]) than mean VTC <10 mg/L. Mean VTCs of 10-15, 15-20, and $>20 \mathrm{mg} / \mathrm{L}$ were not associated with increased hospital mortality (1.154 [0.766-1.739]; 1.342 [0.8962.011]; 1.496 [0.981-2.281]). Similar results were observed in different Acute Physiology and Chronic Health Evaluation IV score or creatinine clearance subgroups.

Conclusions: Increasing mean VTC showed no benefit regarding ICU and hospital mortalities in critically ill patients. Our results suggested that continuous VTC monitoring might not guarantee vancomycin efficacy for ICU patients.

\section{Background}

Vancomycin, a bactericide that acts by obstructing the synthesis of the bacterial cell wall, was isolated from streptomycin approximately half a century ago and exhibits time-dependent bactericidal activity with a long postantibiotic effect [1-4]. Therapeutic drug monitoring (TDM) is an adjuvant and practical method used for vancomycin dosing adjustment in intensive care units (ICUs) because of its narrow therapeutic window [5]. Based on infection models and clinical pharmacokinetic/pharmacodynamic (PK/PD) studies, the area under the concentration-time curve over $24 \mathrm{~h} /$ minimum inhibitory concentration $\left(\mathrm{AUC}_{0-24 \mathrm{~h}} / \mathrm{MIC}\right)$ has been established as the most predictive index to reflect the clinical and microbiological efficacies of vancomycin [6, 7]. Bacterial clearance, along with improvements in clinical signs and symptoms, has been suggested to be associated with $\mathrm{AUC}_{0-24 \mathrm{~h}} / \mathrm{MIC} \geq 400$ [8, 9]. However, it is difficult to precisely determine multiple serum/tissue vancomycin concentrations during the same dosing interval to calculate the AUC in clinical practice [10].

Serum vancomycin trough concentration (VTC) monitoring before the fourth dose has been suggested as the most practical method for TDM of vancomycin, as a VTC $15-20 \mathrm{mg} / \mathrm{L}$ may achieve an $\mathrm{AUC}_{0}-24 \mathrm{~h} / \mathrm{MIC} \geq 400$ if the MIC is $\leq 1 \mathrm{mg} / \mathrm{L}$ [10]. However, plenty of studies have demonstrated that VTC did not have a considerable effect on treatment outcomes $[7,8,11,12]$. Nevertheless, to our knowledge, those most retrospective and prospective studies only focused on the initial VTC after vancomycin therapy. It remains unclear whether the mean VTC, estimated using all measured VTCs during the entire course of therapy, is beneficial for critically ill patients. Therefore, this multicenter observational study with a large sample was further performed to investigate the association of mean VTC with mortality in critically ill patients. 


\section{Patients And Methods}

\section{Study Design and Data Source}

This multicenter observational study was performed using the eICU Collaborative Research Database (eICU-CRD, version 2.0), which is a public de-identified ICU database comprising 200,859 patient unit encounters for 139,367 unique patients admitted between 2014 and 2015. The elCU database is available from

https://physionet.org/content/eicu-crd/. Patients were admitted to 1 of 335 units at 208 hospitals located throughout the United States [13]. The elCU-CRD includes data on vital signs, laboratory measurements, medications, Acute Physiology and Chronic Health Evaluation (APACHE) components, care plan information, admission diagnoses, timestamped diagnoses, and treatments. All researchers of this study received the necessary training and obtained permission to access the database.

\section{Patient Selection}

All patient information was obtained from the elCU-CRD. Adult patients ( $\geq 18$ years) receiving vancomycin therapy with a single hospital admission and two or more TDM records on the first ICU stay were included. The exclusion criteria were as follows: (1) patients with an ICU length of stay $\leq 24 \mathrm{~h}$, (2) patients without records of their ICU discharge status, (3) patients with missing or unqualified covariates for multivariable adjustment, and (4) patients with an outlier value of VTC. The outlier value of VTC was defined when the maximum VTC was greater than 1.5times the interquartile range (IQR) above $\mathrm{Q}_{3}$ or when the minimum VTC was less than 1.5 -times the IQR under $\mathrm{Q}_{1}(\mathrm{IQR}$ was calculated using the formula: $\left.Q_{3}-Q_{1}\right)$.

\section{Outcomes and Covariates}

The outcomes of this study were ICU and hospital mortalities. The variables, mean VTC or serum creatinine (Scr), were calculated by dividing the sum of all collected VTC or Scr by the frequency of monitoring. Mean creatinine clearance (CCl) was determined by the Cockcroft-Gault equation: ([140 - age in years] $\times$ weight in kg / [72 × mean Scr concentration in $\mathrm{mg} / \mathrm{dll}$ ) $\times 0.85$ if female [14]. Related treatments (such as dialysis, ventilation, hypotensor, and hyperensort administration) were included, as these may reflect illness severity and/or affect VTC throughout ICU stay. Variables such as demographic data (e.g., age, sex, and ethnicity), initial body mass index (BMI), APACHE IV score, and comorbidities that could influence mortality (e.g., sepsis, burns, pancreatitis, gastrointestinal bleed, diabetes, heart failure, chronic obstructive pulmonary disease [COPD], hepatic failure, tumor, pneumonia, and renal failure) were also assessed during the first $24 \mathrm{~h}$ of ICU admission.

\section{Statistical Analysis}

Based on the recommended VTC in a series of guidelines $[10,15,16]$, we divided the mean VTC into four categories: $<10,10-15,15-20$, and $>20 \mathrm{mg} / \mathrm{L}$. Continuous variables were presented as the medians (IQRs) and compared using the Kruskal-Wallis $\mathrm{H}$ test. Categorical variables were presented as frequencies (percentages) and compared using $\chi^{2}$ or Fisher's exact tests. A Chord diagram showed the connection between the initial VTC and mean VTC for each patient. Logistic regression models were used to investigate the association of the mean VTC, as a continuous and categorical variable, with ICU and hospital mortalities. To flexibly model and visualize the relationship between mean VTC and mortality, we also used restricted cubic splines with four knots at the $5^{\text {th }}, 35^{\text {th }}, 65^{\text {th }}$, and $95^{\text {th }}$ percentiles. Interaction and subgroup analyses were conducted to determine whether the clinical outcomes persisted when the severity of the clinical status changed or CCl fluctuated. All tests were two-sided, and a $P$ value $<0.05$ was considered statistically significant. Data were extracted using the SAS version 9.4 software (SAS Institute, Cary, NC, USA) and all 
statistical analyses were performed using SPSS 22.0 (SPSS, Inc., Chicago, IL, USA) and Stata 14.0 (Stata Corp., College Station, TX, USA).

\section{Results}

\section{Individual Selection and Clinical Characteristics}

4,256 adult patients with a single hospital stay and at least two VTC records at the first ICU admission were extracted from the eICU-CRD. The following were excluded: 628 patients with a length of ICU stay $\leq 24 \mathrm{~h}, 1$ patient without a record of ICU discharge status, 68 patients with missing or unqualified covariates for multivariable adjustment, and 72 patients with a discrete VTC. Finally, 3,364 patients were included in this study (Fig. 1). The patients were divided into the following four groups according to the mean VTC during ICU stay: $<10 \mathrm{mg} / \mathrm{L}(\mathrm{n}=346,10.3 \%), 10-15 \mathrm{mg} / \mathrm{L}$ ( $\mathrm{n}$ $=1.090,32.4 \%), 15-20 \mathrm{mg} / \mathrm{L}(\mathrm{n}=1,16134.5 \%)$, and $>20 \mathrm{mg} / \mathrm{L}(\mathrm{n}=767,22.8 \%)$. Age, BMI, mean VTC, APACHE IV score, Scr, $\mathrm{CCl}$, and use of hypotensor, hyperensort and dialysis were significantly different among the four groups $(P$ $<0.05)$. Additionally, there was a significant difference in the prevalence of COPD, heart failure, and renal failure $(P<$ 0.05). Groups with a higher mean VTC had higher ICU (4.6\%, 7.7\%, 11.9\%, and $14.7 \%$, respectively; $P<0.001)$ and hospital $(10.7 \%, 14.0 \%, 18.9 \%$, and $21.4 \%$, respectively; $P<0.001)$ mortalities (Table 1$)$. The Chord diagram presented the connection between the mean VTC and initial VTC for each patient (Fig. 2). Among all patients, there were 1,514 (45\%) patients with the mean VTC same as the initial VTC. $230(19.48 \%)$ of patients with initial VTC $<10 \mathrm{mg} / \mathrm{L} \mathrm{had} \mathrm{a}$ mean VTC 15-20 mg/L, Among patients with initial VTC 10-15 mg/L, 439 (44.3\%) patients reached a mean VTC within 15-20 mg/L and 172 (27.4\%) of patients with initial VTC > 20 mg/L had a mean VTC 15-20 mg/L. 
Table 1

Baseline Characteristics of the Study Cohort According to Mean VTC Categories

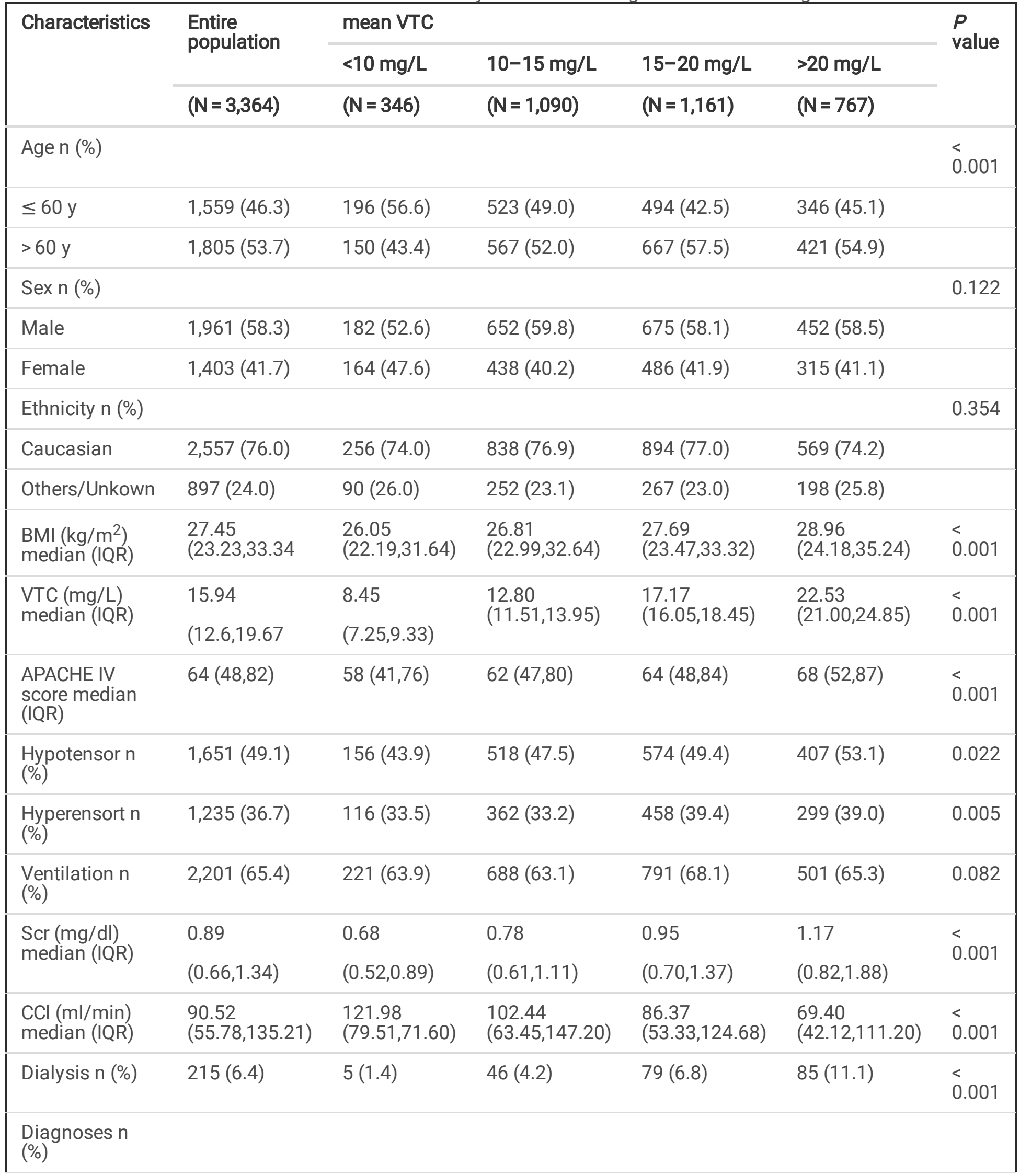

VTC, vancomycin trough concentration; BMI, body mass index; IQR, interquartile range; APACHE, Acute Physiology and Chronic Health Evaluation; Scr, serum creatinine; CCl, creatinine clearance; COPD, chronic obstructive pulmonary disease; ICU, intensive care unit. 


\begin{tabular}{|c|c|c|c|c|c|c|}
\hline \multirow[t]{3}{*}{ Characteristics } & \multirow{2}{*}{$\begin{array}{l}\text { Entire } \\
\text { population }\end{array}$} & \multicolumn{4}{|l|}{ mean VTC } & \multirow{3}{*}{$\begin{array}{l}P \\
\text { value }\end{array}$} \\
\hline & & $<10 \mathrm{mg} / \mathrm{L}$ & $10-15 \mathrm{mg} / \mathrm{L}$ & $15-20 \mathrm{mg} / \mathrm{L}$ & $>20$ mg/L & \\
\hline & $(N=3,364)$ & $(N=346)$ & $(N=1,090)$ & $(\mathrm{N}=1,161)$ & $(N=767)$ & \\
\hline Tumor & $284(8.4)$ & $25(7.2)$ & $91(8.3)$ & $98(8.4)$ & $70(9.1)$ & 0.768 \\
\hline Hepatic failure & $24(0.7)$ & $0(0)$ & $7(0.6)$ & $11(0.9)$ & $6(0.8)$ & 0.313 \\
\hline COPD & $285(8.5)$ & $18(5.2)$ & $100(9.2)$ & $118(10.2)$ & $49(6.4)$ & 0.003 \\
\hline Heart failure & $290(8.6)$ & $13(3.8)$ & $80(7.3)$ & $117(10.1)$ & $80(10.4)$ & $<$ \\
\hline Diabetes & $394(11.7)$ & $36(10.4)$ & $130(11.9)$ & $141(12.1)$ & $87(11.3)$ & 0.818 \\
\hline $\begin{array}{l}\text { Gastrointestinal } \\
\text { bleed }\end{array}$ & $266(7.9)$ & $34(9.8)$ & $81(7.4)$ & $94(8.1)$ & $57(7.4)$ & 0.496 \\
\hline Pancreatitis & $53(1.6)$ & $4(1.2)$ & $18(1.7)$ & $17(1.5)$ & $14(1.8)$ & 0.841 \\
\hline Burns & $8(0.2)$ & $2(0.6)$ & $3(0.3)$ & $3(0.3)$ & $0(0)$ & 0.216 \\
\hline Pneumonia & $884(26.3)$ & $79(22.8)$ & $292(26.8)$ & 319 (27.5) & $194(25.3)$ & 0.319 \\
\hline Sepsis & $1,151(34.2)$ & 105 (30.3) & 363 (33.3) & 419 (36.1) & $264(34.4)$ & 0.210 \\
\hline Renal failure & $479(14.2)$ & $105(30.3)$ & $146(13.4)$ & $178(15.3)$ & $123(16.0)$ & 0.013 \\
\hline $\begin{array}{l}\text { ICU mortality n } \\
(\%)\end{array}$ & 351 (10.4) & $16(4.6)$ & $84(7.7)$ & $138(11.9)$ & $113(14.7)$ & $<0.001$ \\
\hline $\begin{array}{l}\text { Hospital } \\
\text { mortality n (\%) }\end{array}$ & $573(17.0)$ & 37 (10.7) & $153(14.0)$ & 219 (18.9) & $164(21.4)$ & $<$ \\
\hline
\end{tabular}

\section{Association of Mean VTC with Mortality}

The univariable logistic regression model revealed that the mean VTC, as a continuous variable, was positively correlated with ICU (odds ratio, 1.073, 95\% confidence interval, [1.050-1.096]) and hospital (1.052 [1.033-1.070]) mortalities. This association was still robust (1.042 [1.017-1.068]; 1.025 [1.004-1.046], respectively) after adjusting for age, sex, ethnicity, BMI, APACHE IV score, $\mathrm{CCl}$, the use of ventilation, dialysis, hypotensor and hyperensort, and diagnoses at ICU admission (Table 2). When mean VTC was considered as a categorical variable, patients with mean VTCs of 15-20 and > $20 \mathrm{mg} / \mathrm{L}$ were associated with higher ICU (2.782 [1.634-4.738]; 3.564 [2.077-6.115]) and hospital (1.942 [1.339-2.815]; 2.271 [1.550-3.329]) mortalities, and those with mean VTC of 10-15 mg/L showed no significant difference in ICU $(P=0.052)$ and hospital $(P=0.111)$ mortalities compared with those with mean VTC $<10$ $\mathrm{mg} / \mathrm{L}$ in the univariable logistic regression analyses. After multivariable adjustment, mean VTC $10-15 \mathrm{mg} / \mathrm{L}$ was not correlated with ICU mortality $(P=0.160)$, and mean VTCs of $15-20$ and $>20 \mathrm{mg} / \mathrm{L}$ were associated with higher ICU mortality (1.946 [1.106-3.424]; 2.314 [1.296-4.132]) compared with mean VTC of < $10 \mathrm{mg} / \mathrm{L}$. Additionally, the hospital mortality in the groups with mean VTCs of $10-15,15-20$ and $>20 \mathrm{mg} / \mathrm{L}(P=0.494 ; P=0.154 ; P=0.061)$ were not significantly different from those with mean VTC of $<10 \mathrm{mg} / \mathrm{L}$ (Table 2). Restricted cubic splines visually 
showed that the risks of ICU (A) and hospital (B) mortalities increased with an increasing mean VTC (Additional file 1: Fig. 1).

Table 2

Logistic Analysis for the Association of Mean VTC with Mortality

\begin{tabular}{|c|c|c|c|c|}
\hline & ICU Mortality & & Hospital Mortality & \\
\hline VTC Variable & OR $(95 \% \mathrm{Cl})$ & P Value & OR $(95 \% \mathrm{Cl})$ & P Value \\
\hline \multicolumn{5}{|l|}{ Univariable Model } \\
\hline Continuous variable & $1.073(1.050,1.096)$ & $<0.001$ & $1.052(1.033,1.070)$ & $<0.001$ \\
\hline \multicolumn{5}{|l|}{ Categorical variable } \\
\hline$<10 \mathrm{mg} / \mathrm{L}$ & 1 & & 1 & \\
\hline $10-15 \mathrm{mg} / \mathrm{L}$ & $1.722(0.995,2.982)$ & 0.052 & $1.364(0.931,1.997)$ & 0.111 \\
\hline $15-20 \mathrm{mg} / \mathrm{L}$ & $2.782(1.634,4.738)$ & $<0.001$ & $1.942(1.339,2.815)$ & $<0.001$ \\
\hline$>20$ mg/L & $3.564(2.077,6.115)$ & $<0.001$ & $2.271(1.550,3.329)$ & $<0.001$ \\
\hline \multicolumn{5}{|l|}{ Multivariable Model } \\
\hline Continuous variable & $1.042(1.017,1.068)$ & 0.001 & $1.025(1.004,1.046)$ & 0.017 \\
\hline \multicolumn{5}{|l|}{ Categorical variable } \\
\hline$<10 \mathrm{mg} / \mathrm{L}$ & 1 & & 1 & \\
\hline $10-15 \mathrm{mg} / \mathrm{L}$ & $1.512(0.849,2.694)$ & 0.160 & $1.154(0.766,1.739)$ & 0.494 \\
\hline $15-20 \mathrm{mg} / \mathrm{L}$ & $1.946(1.106,3.424)$ & 0.021 & $1.342(0.896,2.011)$ & 0.154 \\
\hline$>20$ mg/L & $2.314(1.296,4.132)$ & 0.005 & $1.496(0.981,2.281)$ & 0.061 \\
\hline \multicolumn{5}{|c|}{$\begin{array}{l}\text { Multivariable model: adjusted for age (category), sex, ethnicity, BMI, APACHE IV score, CCI, the use of ventilation, } \\
\text { dialysis, hypotensor and hyperensort, and diagnoses at ICU admission (tumor, hepatic failure, COPD, heart failure, } \\
\text { diabetes, gastrointestinal bleed, pancreatitis, burns, pneumonia, sepsis, and renal failure) } \\
\text { VTC, vancomycin trough concentration; ICU, intensive care unit; OR, odds ratio; Cl, confidence interval; BMI, body } \\
\text { mass index; APACHE, Acute Physiology and Chronic Health Evaluation; CCl, creatinine clearance; COPD, chronic } \\
\text { obstructive pulmonary disease. }\end{array}$} \\
\hline
\end{tabular}

\section{Association of Mean VTC with Mortality in Different Subgroups}

We further analyzed the association between mean VTC and mortality in different predefined subgroups: APACHE IV score $\leq 64(\mathrm{n}=1,736)$ and APACHE IV score $>64(\mathrm{n}=1,628)$ or $\mathrm{CCl} \leq 80 \mathrm{ml} / \mathrm{min}(\mathrm{n}=1,460)$ and CCl $>80 \mathrm{ml} / \mathrm{min}(\mathrm{n}=$ 1,904). We found no interaction between APACHE IV score (category) and mean VTC (ICU mortality, $P_{\text {interaction }}=$ 0.721 ; hospital mortality, $\left.P_{\text {interaction }}=0.067\right)$. Similarly, no interaction was found between mean VTC and CCI for ICU $\left(P_{\text {interaction }}=0.807\right)$ and hospital $\left(P_{\text {interaction }}=0.756\right)$ mortalities. Notably, the multivariable logistical regression results were still robust across different subgroups. We observed that mean VTC, as a continuous variable, was significantly correlated with both ICU and hospital mortalities in APACHE IV score $>64(P<0.001 ; P<0.001)$ and CCI $\leq 80 \mathrm{ml} / \mathrm{min}(P=0.017 ; P=0.002)$ subgroups. When mean VTC was used as a categorical variable, the groups with mean VTCs of $15-20$ and $>20 \mathrm{mg} / \mathrm{L}$ exhibited a statistical difference in ICU $(P=0.041 ; P=0.005)$ and hospital $(P=$ 0.017; $P=0.003$ ) mortalities compared to those with mean VTC $<10 \mathrm{mg} / \mathrm{L}$ in APACHE IV score $>64$ subgroups. 
Similarly, mean VTC $>20 \mathrm{mg} / \mathrm{L}$ was an independent risk factor for ICU mortality $(P=0.012)$ in $\mathrm{CCl} \leq 80 \mathrm{ml} / \mathrm{min}$ subgroup (Table 3; Table 4).

Table 3

Multivariable Analysis for Association of Mean VTC with Mortality in APACHE IV Score Subgroups

\begin{tabular}{|c|c|c|c|c|}
\hline & ICU Mortality & & Hospital Mortality & \\
\hline VTC Variable & OR (95\% Cl) & P Value & OR (95\% Cl) & P Value \\
\hline \multicolumn{5}{|l|}{ APACHE IV score $\leq 64$} \\
\hline Continuous variable & $1.001(0.957,1.048)$ & 0.958 & $0.979(0.944,1.015)$ & 0.241 \\
\hline \multicolumn{5}{|l|}{ Categorical variable } \\
\hline$<10 \mathrm{mg} / \mathrm{L}$ & 1 & & 1 & \\
\hline $10-15 \mathrm{mg} / \mathrm{L}$ & $1.268(0.496,3.245)$ & 0.620 & $0.801(0.441,1.456)$ & 0.467 \\
\hline $15-20 \mathrm{mg} / \mathrm{L}$ & $1.486(0.591,3.737)$ & 0.400 & $0.798(0.440,1.448)$ & 0.459 \\
\hline$>20$ mg/L & $1.458(0.545,3.896)$ & 0.452 & $0.772(0.399,1.491)$ & 0.441 \\
\hline \multicolumn{5}{|l|}{ APACHE IV score > 64} \\
\hline Continuous variable & $1.061(1.030,1.092)$ & $<0.001$ & $1.049(1.024,1.075)$ & $<0.001$ \\
\hline \multicolumn{5}{|l|}{ Categorical variable } \\
\hline$<10 \mathrm{mg} / \mathrm{L}$ & 1 & & 1 & \\
\hline $10-15 \mathrm{mg} / \mathrm{L}$ & $1.589(0.770,3.278)$ & 0.210 & $1.534(0.882,2.667)$ & 0.130 \\
\hline $15-20 \mathrm{mg} / \mathrm{L}$ & $2.095(1.032,4.254)$ & 0.041 & $1.944(1.128,3.349)$ & 0.017 \\
\hline$>20 \mathrm{mg} / \mathrm{L}$ & $2.770(1.350,5.683)$ & 0.005 & $2.304(1.319,4.025)$ & 0.003 \\
\hline \multicolumn{5}{|c|}{$\begin{array}{l}\text { Multivariable model: adjusted for age (category), sex, ethnicity, BMI, CCl, the use of ventilation, dialysis, } \\
\text { hypotensor and hyperensort, and diagnoses at ICU admission (tumor, hepatic failure, COPD, heart failure, diabetes, } \\
\text { gastrointestinal bleed, pancreatitis, burns, pneumonia, sepsis, and renal failure) } \\
\text { VTC, vancomycin trough concentration; APACHE, Acute Physiology and Chronic Health Evaluation; ICU, intensive } \\
\text { care unit; OR, odds ratio; Cl, confidence interval; BMI, body mass index; CCl, creatinine clearance; COPD, chronic } \\
\text { obstructive pulmonary disease. }\end{array}$} \\
\hline
\end{tabular}


Table 4

Multivariable Analysis for Association of Mean VTC with Mortality in CCI Subgroups

\begin{tabular}{|c|c|c|c|c|}
\hline & ICU Mortality & & Hospital Mortality & \\
\hline VTC Variable & OR $(95 \% \mathrm{Cl})$ & $P$ Value & OR $(95 \% \mathrm{Cl})$ & $P$ Value \\
\hline \multicolumn{5}{|l|}{$\mathrm{CCl} \leq 80 \mathrm{ml} / \mathrm{min}$} \\
\hline Continuous variable & $1.049(1.018,1.082)$ & 0.002 & $1.032(1.006,1.060)$ & 0.017 \\
\hline \multicolumn{5}{|l|}{ Categorical variable } \\
\hline$<10 \mathrm{mg} / \mathrm{L}$ & 1 & & 1 & \\
\hline $10-15 \mathrm{mg} / \mathrm{L}$ & $1.612(0.706,3.683)$ & 0.257 & $0.986(0.541,1.797)$ & 0.963 \\
\hline $15-20 \mathrm{mg} / \mathrm{L}$ & $2.133(0.956,4.758)$ & 0.064 & $1.295(0.724,2.317)$ & 0.384 \\
\hline$>20 \mathrm{mg} / \mathrm{L}$ & $2.813(1.251,6.325)$ & 0.012 & $1.461(0.807,2.643)$ & 0.211 \\
\hline \multicolumn{5}{|l|}{$\mathrm{CCl}>80 \mathrm{ml} / \mathrm{min}$} \\
\hline Continuous variable & $1.035(0.993,1.079)$ & 0.100 & $1.016(0.984,1.049)$ & 0.335 \\
\hline \multicolumn{5}{|l|}{ Categorical variable } \\
\hline$<10 \mathrm{mg} / \mathrm{L}$ & 1 & & 1 & \\
\hline $10-15 \mathrm{mg} / \mathrm{L}$ & $1.437(0.637,3.237)$ & 0.382 & $1.330(0.755,2.344)$ & 0.323 \\
\hline 15-20 mg/L & $1.905(0.8541,4.248)$ & 0.115 & $1.383(0.782,2.446)$ & 0.265 \\
\hline$>20$ mg/L & $1.800(0.757,4.282)$ & 0.184 & $1.465(0.784,2.738)$ & 0.231 \\
\hline \multicolumn{5}{|c|}{$\begin{array}{l}\text { Multivariable model: adjusted for age (category), sex, ethnicity, BMI, APACHE IV score, the use of ventilation, } \\
\text { dialysis, hypotensor and hyperensort, and diagnoses at ICU admission (tumor, hepatic failure, COPD, heart failure, } \\
\text { diabetes, gastrointestinal bleed, pancreatitis, burns, pneumonia, sepsis, and renal failure) } \\
\text { VTC, vancomycin trough concentration; CCI, creatinine clearance; ICU, intensive care unit; OR, odds ratio; Cl, } \\
\text { confidence interval; BMl, body mass index; APACHE, Acute Physiology and Chronic Health Evaluation; COPD, } \\
\text { chronic obstructive pulmonary disease. }\end{array}$} \\
\hline
\end{tabular}

\section{Discussion}

In this retrospective multicenter cohort study, we recruited a total of 3,364 critically ill patients with two or more VTC monitoring records after vancomycin therapy from 335 different ICUs at 208 hospitals in the elCU-CRD. Mean VTC was calculated by dividing the sum of all collected VTC by the frequency of monitoring for each patient. Our study showed that mean VTCs of 10-15, 15-20 and $>20 \mathrm{mg} / \mathrm{L}$ failed to reduce the ICU and hospital mortalities for critically ill patients. Patients with mean VTCs of $15-20$ and $>20 \mathrm{mg} / \mathrm{L}$ were found to be exposed to even higher risks of ICU mortality. The results indicated that maintaining high serum VTC throughout the entire course of vancomycin therapy failed to demonstrate benefit for ICU and hospital mortalities in critically ill patients.

As serum VTC has been suggested as a surrogate marker for the AUC/MIC index to monitor vancomycin efficacy, several studies have been designed to verify the association of VTC with clinical and microbiological outcomes in critically ill patients [10]. Surprisingly, most studies found no statistical difference in treatment outcomes according to the VTC level. Two retrospective studies have shown that VTC alone is not a good indicator for vancomycin treatment success among patients with methicillin-resistant Staphylococcus aureus (MRSA) bacteremia [7, 8]. Two other 
retrospective cohort studies have demonstrated that VTC $>15 \mathrm{mg} / \mathrm{L}$ fails to improve the outcomes of patients with MRSA infection $[17,18]$. Additionally, two prospective, multicenter, observational studies have demonstrated no significant association between VTC level and vancomycin treatment response in a Chinese population diagnosed with gram-positive bacterial infections $[11,19]$. Moreover, the latest consensus suggests that there are minimal to no data to support the safety and efficacy of a target VTC of 15-20 mg/L in patients with serious MRSA infections [9]. However, those aforementioned studies only explored the relationship between single steady-state VTC and outcomes. In a retrospective study of 76 critically ill patients confirmed MRSA infections, Cheong reported that the initial VTC was not associated with treatment response, which was consistent with the results of the other studies. Surprisingly, a corrected VTC, calculated as dividing the sum of each measured VTC multiplied by the number of days at that level by the total number of days under treatment, was higher among patients with improved clinical presentation and laboratory results than among those with poor clinical outcomes [20]. Because of the lack of largescale population studies on multiple VTC records after receiving vancomycin therapy, which may represent personalized PK/PD profiles of vancomycin, whether the mean VTC derived from the entire course of therapy is of potential benefit for critically ill patients remains unclear.

In this study, the mean VTC was estimated using all collected VTCs throughout the therapy course, providing an overall level of VTC during the ICU stay. The chord diagram showed initial VTC and mean VTC was inconsistent in $55 \%$ of all patients. $19.48 \%$ of patients with initial VTC $<10 \mathrm{mg} / \mathrm{L}, 44.3 \%$ of patients with initial VTC $10-15 \mathrm{mg} / \mathrm{L}$, and $27.4 \%$ of patients with initial VTC $>20 \mathrm{mg} / \mathrm{L}$, had reached a mean VTC 15-20 mg/L eventually, which indicated that initial subtherapeutic or excessive VTC had been adjusted to achieve suggested VTC. Therefore, mean VTC could reflect exposure dosage of vancomycin after adjustment for those with lower or higher initial VTC, to some extent. We further found that mean VTC of 15-20 and > $20 \mathrm{mg} / \mathrm{L}$ were significantly correlated with higher ICU mortality (1.946fold and 2.314-fold) than a mean VTC of $<10 \mathrm{mg} / \mathrm{L}$. Moreover, mean VTCs of 10-15 and 15-20 mg/L had no benefit for hospital mortality. Therefore, this present study is one in a growing number of studies demonstrating that maintaining suggested VTC might not ensure vancomycin efficacy for critically ill patients.

The APACHE IV score is useful for assessing the severity of illness and predicting outcomes in ICU patients [21, 22]. To diminish the influence of disease severity itself, all individuals were divided into two subgroups for further investigations based on the median first APACHE IV score ( $\leq 64$ or $>64$ ). Mean VTCs of $10-15$ and $15-20 \mathrm{mg} / \mathrm{L}$ might not offer a survival advantage in APACHE IV score $\leq 64$ subgroup. In APACHE IV score > 64 subgroup, mean VTCs of 10-15 and 15-20 mg/L were risk factors for ICU and hospital mortalities. Therefore, regardless of the severity of critically ill patients, high mean VTC might not be beneficial for patients receiving the vancomycin.

Vancomycin is eliminated primarily via the renal route, with $>80-90 \%$ recovered unchanged in urine within $24 \mathrm{~h}$ after administration of a single dose [4]. A decrease in the glomerular filtration rate for any cause would increase the VTC, making the association between mortality and VTC difficult to assess [23]. To exclude the influence of renal function on outcomes, we classified the patients into two groups according to $\mathrm{CCl}$ ( $\leq 80 \mathrm{or}>80 \mathrm{ml} / \mathrm{min}$ ) to assess this relationship. The results showed that mean VTCs of 10-15 and 15-20 mg/L failed to reduce ICU and hospital mortalities for ICU patients in both the $\mathrm{CCl}>80$ and $\leq 80 \mathrm{ml} / \mathrm{min}$ subgroups. Moreover, a mean VTC of $>20 \mathrm{mg} / \mathrm{L}$ might serve as an independent risk factor for ICU mortality in the $\mathrm{CCl} \leq 80 \mathrm{ml} / \mathrm{min}$ subgroup. Based on these results, other index of TDM for vancomycin management, such as AUC/MIC or Bayesian AUC-only estimation, should be considered to improve prognosis.

A few underlying mechanisms may explain our results. First, Patel et al. reported that patients with a VTC of 15-20 $\mathrm{mg} / \mathrm{L}$ could achieve an $\mathrm{AUC}_{0-24} / \mathrm{MIC}$ ratio of $\geq 400$ when the MIC value is $\leq 1$ [24]. However, several studies have demonstrated a poor correlation between VTC and $\mathrm{AUC}_{0-24}$ because of high inter-individual variability [25-28]. A 3- 
year, prospective study indicated that $68 \%$ of adults who were administered vancomycin with an $\mathrm{AUC}_{0-24} \geq 400$ $\mathrm{mg} \cdot \mathrm{h} / \mathrm{L}$ had a trough concentration of $<15 \mathrm{mg} / \mathrm{L}$ [29]. Additionally, a prospective study of Chinese patients suggested that $\mathrm{C}_{\text {max }}, \mathrm{AUC}_{0-24}$, and $\mathrm{AUC}_{0-24 \mathrm{~h}} / \mathrm{MIC}$ are not significantly associated with clinical and microbiological outcomes based on multivariable logistic regression analysis [19]. Second, vancomycin has been associated with several adverse events. A multicenter prospective clinical trial that included 288 adult patients indicated that a VTC of $>15$ $\mathrm{mg} / \mathrm{L}$ is associated with a 3 -fold increased risk of nephrotoxicity [30]. Another prospective multicenter clinical study in Chinese patients observed that vancomycin nephrotoxicity is significantly correlated with its trough concentration and the cut-off is $13 \mathrm{mg} / \mathrm{L}$ [11]. The optimal TDM of vancomycin in ICU patients warrants further investigation.

\section{Limitations}

Our study has some limitations. First, given the retrospective nature of the study, the risk of unmeasured confounding effects and introduction of bias were unavoidable. However, this multicenter study of VTC included the largest sample size to date, allowing the findings to be generalized. Second, there is no available information on microbiological outcomes after vancomycin treatment in eICU-CRD. Nevertheless, mortality may represent clinical outcomes to confirm the prognostic value of VTC, as critically ill patients with gram-positive bacterial infections have a high mortality, particularly owing to MRSA infection [31]. Third, we can provide only the association between mean VTC and mortality rather than causality. In the future, a well-designed randomized controlled trial should be considered to evaluate causality between VTC and mortality.

\section{Conclusions}

In conclusion, increasing mean serum VTC showed no benefit for ICU and hospital mortalities in critically ill patients, independent of the disease severity or renal function. Therefore, our results suggested that continuous VTC monitoring might not ensure vancomycin efficacy for ICU patients. TDM for vancomycin management using AUC/MIC or Bayesian AUC-only estimation should be studied in the future.

\section{Abbreviations}

TDM, therapeutic drug monitoring; ICU, intensive care unit; PK/PD, pharmacokinetic/pharmacodynamics; AUC024h/MIC, area under the concentration-time curve over 24 hours/minimum inhibitory concentration; VTC, vancomycin trough concentration; eICU-CRD, elCU Collaborative Research Database; APACHE, Acute Physiology and Chronic Health Evaluation; IQR, interquartile range; Scr, serum creatinine; $\mathrm{CCl}$, creatinine clearance; BMI, body mass index; COPD, chronic obstructive pulmonary disease; MRSA, methicillin-resistant Staphylococcus aureus.

\section{Declarations}

\section{Ethical approval and consent to participate[}

As all protected patient health-related information in the elCU had been deleted, the requirement for individual patient consent was waived. The database is released under the Health Insurance Portability and Accountability Act (HIPAA) safe harbor provision. The re-identification risk was certified as meeting safe harbor standards by Privacert (Cambridge, MA) (HIPAA Certification no. 1031219-2).

\section{Consent for publication:}

Not applicable. 
Availability of data and materials:

The datasets generated and analyzed during the current study is available in the elCU repository, https://doi.org/10.13026/C2WM1R[13].

\section{Competing interests:}

The authors declare that they have no competing interests.

\section{Funding:}

This study was supported by the National Natural Science Foundation of China (81770057).

\section{Author contributions:}

GW, YH, and JR conceived of the study. GW provided funding for the study. GW, XL and XW provided critical appraisal and revision. JL, RL, YG, XJ, JZ, and JR extracted and collected data from elCU-CRD. YH, XJ, and JL performed statistical analysis. YH and JR wrote the manuscript. $Y H, J R, J L, X J, Y G, R L$, and JZ revised the manuscript. All authors read and approved the final manuscript.

\section{Acknowledgements:}

We appreciate the researchers at the MIT Laboratory for Computational Physiology and collaborating research groups to keep eICU-CRD databases available.

\section{References}

1. Moellering RC, Jr. Vancomycin: a 50-year reassessment. Clin Infect Dis 2006;42 Suppl 1:S3-4.

2. Liu C, Bayer A, Cosgrove SE, et al. Clinical practice guidelines by the infectious diseases society of america for the treatment of methicillin-resistant Staphylococcus aureus infections in adults and children. Clin Infect Dis 2011;52:e18-55.

3. Álvarez R, López Cortés LE, Molina J, et al. Optimizing the Clinical Use of Vancomycin. Antimicrob Agents Chemother 2016;60:2601-9.

4. Rybak MJ. The pharmacokinetic and pharmacodynamic properties of vancomycin. Clin Infect Dis 2006;42 Suppl 1:S35-9.

5. Tabah A, De Waele J, Lipman J, et al. The ADMIN-ICU survey: a survey on antimicrobial dosing and monitoring in ICUs. J Antimicrob Chemother 2015;70:2671-7.

6. Moise-Broder PA, Forrest A, Birmingham MC, et al. Pharmacodynamics of vancomycin and other antimicrobials in patients with Staphylococcus aureus lower respiratory tract infections. Clin Pharmacokinet 2004;43:925-42.

7. Jung $\mathrm{Y}$, Song $\mathrm{KH}$, Cho J, et al. Area under the concentration-time curve to minimum inhibitory concentration ratio as a predictor of vancomycin treatment outcome in methicillin-resistant Staphylococcus aureus bacteraemia. Int J Antimicrob Agents 2014;43:179-83.

8. Makmor-Bakry M, Ahmat A, Shamsuddin A, et al. Association between single trough-based area under the curve estimation of vancomycin and treatment outcome among methicillin-resistant Staphylococcus aureus bacteremia patients. Anaesthesiol Intensive Ther 2019;51:218-23. 
9. Rybak MJ, Le J, Lodise TP, et al. Therapeutic monitoring of vancomycin for serious methicillin-resistant Staphylococcus aureus infections: A revised consensus guideline and review by the American Society of HealthSystem Pharmacists, the Infectious Diseases Society of America, the Pediatric Infectious Diseases Society, and the Society of Infectious Diseases Pharmacists. Am J Health Syst Pharm 2020;77:835-64.

10. Rybak M, Lomaestro B, Rotschafer JC, et al. Therapeutic monitoring of vancomycin in adult patients: a consensus review of the American Society of Health-System Pharmacists, the Infectious Diseases Society of America, and the Society of Infectious Diseases Pharmacists. Am J Health Syst Pharm 2009;66:82-98.

11. Liang X, Fan Y, Yang M, et al. A Prospective Multicenter Clinical Observational Study on Vancomycin Efficiency and Safety With Therapeutic Drug Monitoring. Clin Infect Dis 2018;67:S249-s55.

12. Albur MS, Bowker $\mathrm{K}$, Weir I, et al. Factors influencing the clinical outcome of methicillin-resistant Staphylococcus aureus bacteraemia. Eur J Clin Microbiol Infect Dis 2012;31:295-301.

13. Pollard TJ, Johnson AEW, Raffa JD, et al. The elCU Collaborative Research Database, a freely available multicenter database for critical care research. Sci Data 2018;5:180178.

14. Cockcroft DW, Gault MH. Prediction of creatinine clearance from serum creatinine. Nephron 1976;16:31-41.

15. Ye ZK, Chen YL, Chen K, et al. Therapeutic drug monitoring of vancomycin: a guideline of the Division of Therapeutic Drug Monitoring, Chinese Pharmacological Society. J Antimicrob Chemother 2016;71:3020-5.

16. Matsumoto K, Takesue Y, Ohmagari N, et al. Practice guidelines for therapeutic drug monitoring of vancomycin: a consensus review of the Japanese Society of Chemotherapy and the Japanese Society of Therapeutic Drug Monitoring. J Infect Chemother 2013;19:365-80.

17. Clemens EC, Chan JD, Lynch JB, et al. Relationships between vancomycin minimum inhibitory concentration, dosing strategies, and outcomes in methicillin-resistant Staphylococcus aureus bacteremia. Diagn Microbiol Infect Dis 2011;71:408-14.

18. Hall RG, 2nd, Blaszczyk AT, Thompson KA, et al. Impact of empiric weight-based vancomycin dosing on nephrotoxicity and mortality in geriatric patients with methicillin-resistant Staphylococcus aureus bacteraemia. J Clin Pharm Ther 2014;39:653-7.

19. Shen K, Yang M, Fan Y, et al. Model-based Evaluation of the Clinical and Microbiological Efficacy of Vancomycin: A Prospective Study of Chinese Adult In-house Patients. Clin Infect Dis 2018;67:S256-s62.

20. Cheong JY, Makmor-Bakry M, Lau CL, et al. The relationship between trough concentration of vancomycin and effect on methicillin-resistant Staphylococcus aureus in critically ill patients. S Afr Med J 2012;102:616-9.

21. Zimmerman JE, Kramer AA, McNair DS, et al. Acute Physiology and Chronic Health Evaluation (APACHE) IV: hospital mortality assessment for today's critically ill patients. Crit Care Med 2006;34:1297-310.

22. Ko M, Shim M, Lee SM, et al. Performance of APACHE IV in Medical Intensive Care Unit Patients: Comparisons with APACHE II, SAPS 3, and MPM(0) III. Acute Crit Care 2018;33:216-21.

23. Cantú TG, Yamanaka-Yuen NA, Lietman PS. Serum vancomycin concentrations: reappraisal of their clinical value. Clin Infect Dis 1994;18:533-43.

24. Patel N, Pai MP, Rodvold KA, et al. Vancomycin: we can't get there from here. Clin Infect Dis 2011;52:969-74.

25. Bakke V, Sporsem H, Von der Lippe E, et al. Vancomycin levels are frequently subtherapeutic in critically ill patients: a prospective observational study. Acta Anaesthesiol Scand 2017;61:627-35.

26. Neely MN, Youn G, Jones B, et al. Are vancomycin trough concentrations adequate for optimal dosing? Antimicrob Agents Chemother 2014;58:309-16.

27. Tsai D, Stewart PC, Hewagama S, et al. Optimised dosing of vancomycin in critically ill Indigenous Australian patients with severe sepsis. Anaesth Intensive Care 2018;46:374-80. 
28. Haeseker M, Croes S, Neef C, et al. Evaluation of Vancomycin Prediction Methods Based on Estimated Creatinine Clearance or Trough Levels. Ther Drug Monit 2016;38:120-6.

29. Neely MN, Kato L, Youn G, et al. Prospective Trial on the Use of Trough Concentration versus Area under the Curve To Determine Therapeutic Vancomycin Dosing. Antimicrob Agents Chemother 2018;62.

30. Bosso JA, Nappi J, Rudisill C, et al. Relationship between vancomycin trough concentrations and nephrotoxicity: a prospective multicenter trial. Antimicrob Agents Chemother 2011;55:5475-9.

31. Calfee DP, Salgado CD, Milstone AM, et al. Strategies to prevent methicillin-resistant Staphylococcus aureus transmission and infection in acute care hospitals: 2014 update. Infect Control Hosp Epidemiol 2014;35 Suppl 2:S108-32.

\section{Figures}




\section{4,256 patients from eICU-CRD (Version 2.0):}

1. With a single hospital admission;

2. With aged $\geq 18$ years;

3. With vancomycin therapy and two or more TDM

Patients with ICU length of stay $\leq 24$

hours

$(\mathrm{N}=628)$

Patients with no record of ICU

discharge status

$(\mathrm{N}=1)$

Patients with missing or unqualified

covariates for multivariable adjustment

$(\mathrm{N}=68)$

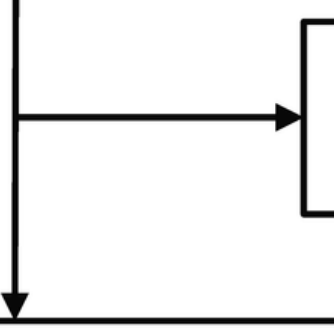

\section{Patients with discrete value of VTC}

$(\mathrm{N}=72$ )

\section{3,364 patients}

Figure 1

Flow chart of participant selection. Cohort selection and criteria for exclusion, a total of 3,364 patients were included in the analysis. eICU-CRD, eICU Collaborative Research Database; ICU, intensive care unit; TDM, therapeutic drug monitoring; VTC, vancomycin trough concentration. 


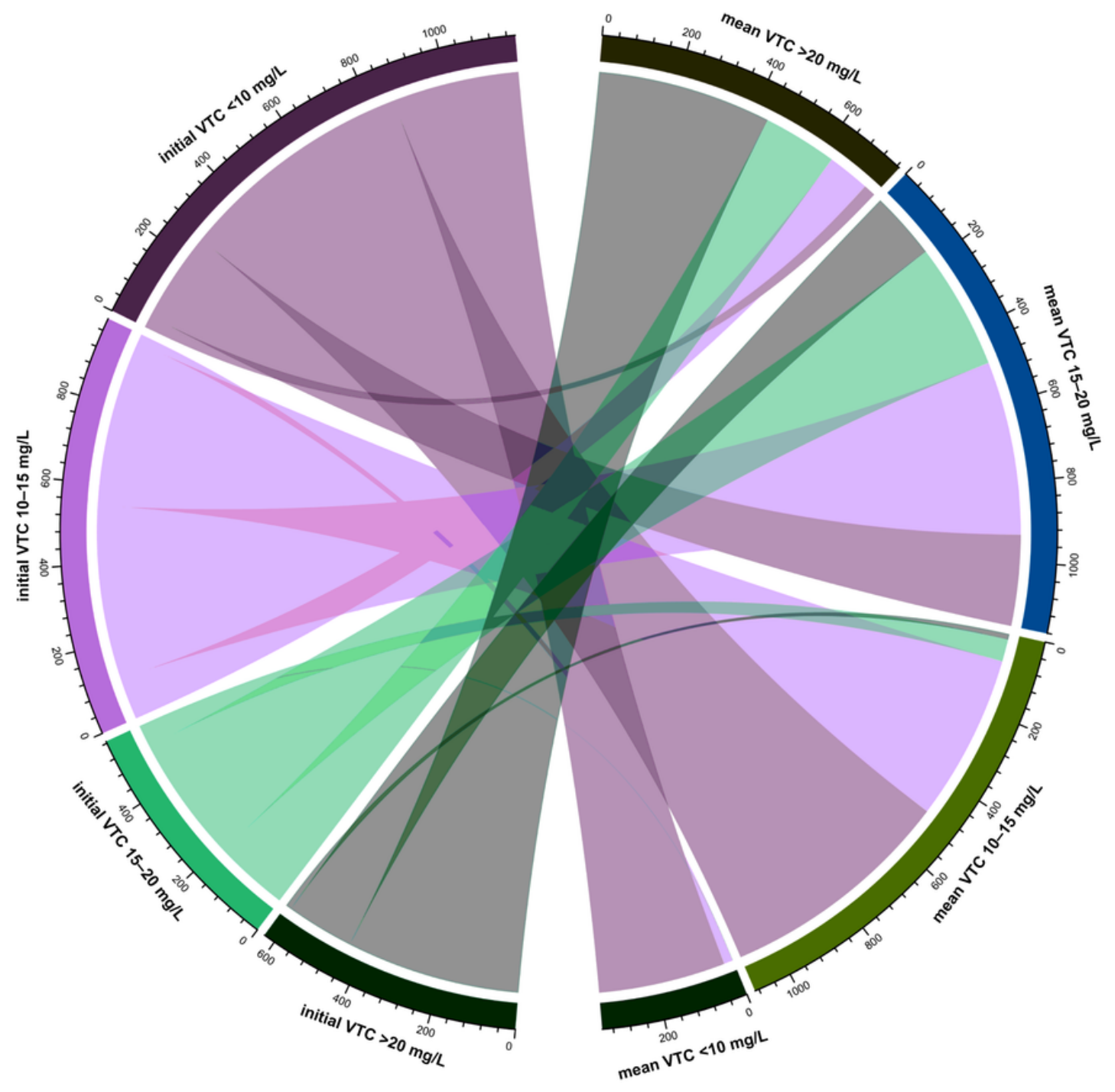

Figure 2

Connection between the mean VTC and initial VTC. A chord diagram presented the difference of the mean VTC with initial VTC for each patient. VTC, vancomycin trough concentration

\section{Supplementary Files}

This is a list of supplementary files associated with this preprint. Click to download.

- AdditionalFile.doc

- figures1.tif 